\title{
umcG \\ Effectiveness and complications in the use of ultrasound guidance for vascular cannulation among 3rd year residents.
}

B Armocida , RH Gestel, JKG Wietasch, T.W.L. Scheeren, M Modestini Dept. of Anesthesiology, University of Groningen, University Medical Center Groningen,

Groningen, The Netherlands

Background: In the literature there is controversy regarding the effectiveness of ultrasound guidance during peri-operative vascular cannulation ${ }^{1}$. We observed among third year anaesthesia residents if the use of an ultrasound (US) guided technique for arterial and jugular venous catheterization leads to faster insertion times, higher success rates and fewer complications compared to the blind palpation or landmark technique.

Methods: With approval of local medical ethic committee we observed the insertion by third year residents of the radial artery catheter and central venous catheter (CVC) in patients undergoing cardiac surgery. The residents were free to choose the cannulation technique. The study endpoints were the success rate considered as numbers of attempts (each attempt being defined as a new skin puncture), time required for insertion and the immediate complications (ischemia, bleeding, hematoma, pneumothorax and vasospasm).
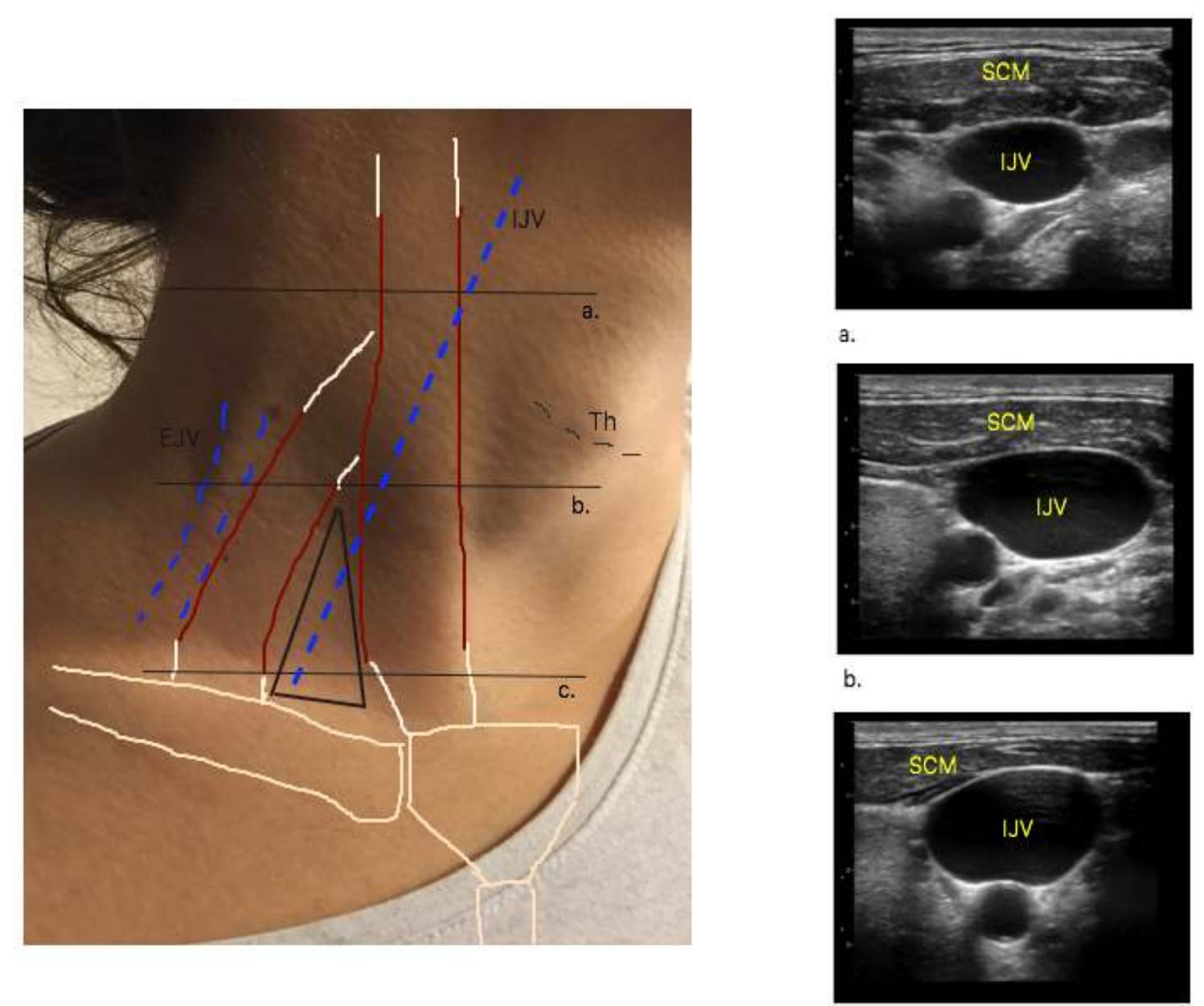

Fig.1 Sternocleido mastoid muscle (SCM) with its sternal and clavicular head. Internal jugular vein (IJV). External jugular vein (EJV). Thyroid (Th). Sedillot's triangle is formed by the sternal head and the clavicular head of SCM and clavicle.
Results: A total of 34 patients and six residents were included in the study. The arterial catheterization was successful on the first attempt in $9 / 11$ patients in the US guided group and in 21/23 patients in the palpation group. The mean insertion time was 2,6 vs. $2,1 \mathrm{~min}$, respectively. Two complications occurred with the palpation technique, 1 with the US guided one. The CVC was inserted on the first attempt in $16 / 16$ patients in the US group and in 12/18 patients in the landmark group. Overall, 6 complications ( 3 arterial punctures and 3 local hematomas) occurred in the landmark technique group, while no complication occurred when US was used $(p<0.05)$.

Conclusion: For radial arterial catheterization the use of US did not improve clinical practice compared to blind palpation among 3rd year anaesthesia residents. On the contrary for central venous access US guided cannulation was associated with reduced complications and increased success rate compared to blind palpation or landmark technique.

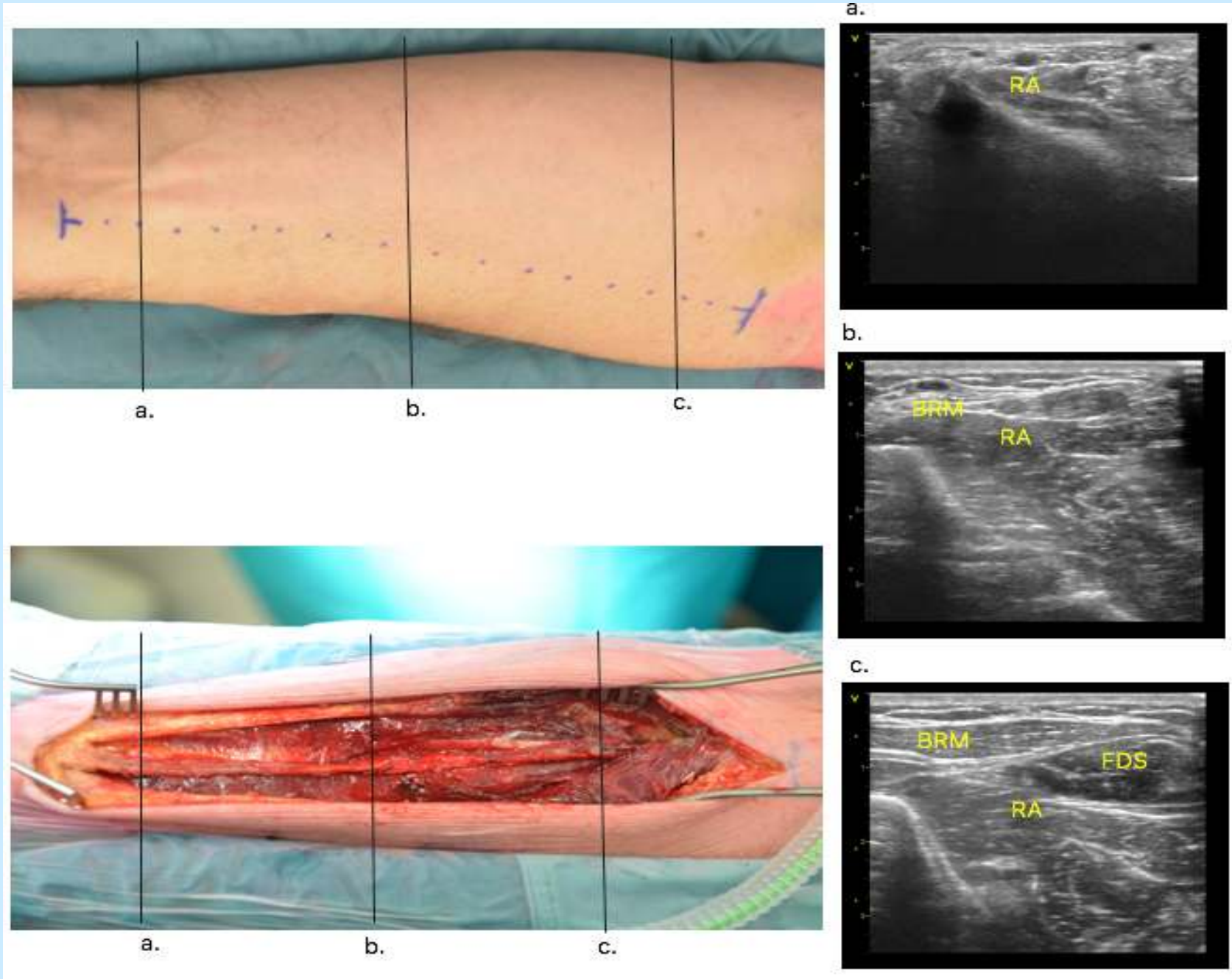

Fig.2 (a) In the distal forearm the radial artery (RA) is more superficial and well visible. (b) In the medial forearm RA begins to go deep in brachioradialis muscle (BRM). (c) In the proximal forearm RA goes under brachioradialis muscle (BRM) and flexor digitorum sublimis muscle (FDS). 